"C 2020 IEEE. Personal use of this material is permitted. Permission from IEEE must be obtained for all other uses, in any current or future media, including reprinting/republishing this material for advertising or promotional purposes, creating new collective works, for resale or redistribution to servers or lists, or reuse of any copyrighted component of this work in other works." 


\title{
Communication Improved Beam-Scannable Ultra-Wideband Sparse Antenna Arrays by Iterative Convex Optimization Based on Raised Power Series Representation
}

\author{
Yanhui Liu, Senior Member, IEEE, Yuqi Yang, Feng Han, Qing Huo Liu, Fellow, IEEE, and Y. Jay Guo, Fellow, IEEE
}

\begin{abstract}
A novel method is presented to design beam-scannable ultra-wideband (UWB) sparse arrays. A concept of design frequency is introduced which transforms the beam-scannable UWB array design to the problem of synthesizing a broadside-beam array at single-frequency. The raised power series (RPS) representation with appropriate parameter selection is adopted to generate initial element positions, and then an iterative convex optimization is applied to successively optimize the element positions for further sidelobe level (SLL) reduction. Multiple constraints for controlling the first-order Taylor expansion accuracy, the minimum element spacing and the array aperture are incorporated in the iterative convex optimization to obtain stable and practical synthesis results. Several examples for synthesizing UWB arrays with different frequency bands, beam scanning ranges and element counts, are conducted to validate the effectiveness and advantages of the proposed method. It is shown that the proposed method achieves much lower SLLs than those by the original RPS method for all test cases, and it also significantly outperforms some conventional stochastic optimization methods for large UWB array cases.
\end{abstract}

Index Terms-Ultra-wideband (UWB) sparse array, beam-scannable array, convex optimization, raised power series (RPS), minimum element spacing control.

\section{INTRODUCTION}

$\mathbf{U}$ LTRA-wideband (UWB) antenna arrays are of great importance in many applications such as passive radars, microwave imaging systems, high-speed communications and radio astronomy [1], [2]. For the UWB array, the highest-to-lowest frequency ratio (HLFR) can reach $3: 1$ or even more. In this situation, the design of UWB arrays is much more difficult than that in the narrow-band case. One of the key challenging problems is to choose element positions for UWB arrays. In particular, when considering uniformly spaced UWB arrays with wide-angle beam-scanning capability, designers would face a dilemma that, to avoid the presence of grating lobes in the whole interested band, the element spacing should be no larger than half a wavelength at the highest frequency, while to enable the antenna elements to radiate effectively with good impedance matching, a spacing of half a wavelength at the lowest frequency would be usually required. There are two ways to deal with this problem. One way is choosing small element spacing to eliminate grating lobes at highest frequency and applying some sophisticated techniques such as tightly coupled antenna array design to enhance the radiation and matching performance of antenna elements at low frequency [3], [4]. This kind of UWB arrays have the advantage of high aperture efficiency, but

Manuscript received xxx. This work was supported in part by the Natural Science Foundation of China (NSFC) under Grant No. 61871338, in part by the Science and Technology Research Project of Fujian Province under Grant No 2017I0017, in part by the Joint Fund of Ministry of Education under Grant No. 6141A02033338, and in part by the University of Technology Sydney. (corresponding email: yanhuiliu@xmu.edu.cn)

Y. Liu, Y. Yang, and F. Han are with Institute of Electromagnetics and Acoustics, Xiamen University, Fujian 361005, China. Y. Liu is also with the Global Big Data Technologies Centre, University of Technology Sydney (UTS), NSW 2007, Australia

Q. H. Liu is with the Department of Electrical and Computer Engineering, Duke University, Durham, NC 27708, USA.

Y. Jay Guo is with the Global Big Data Technologies Centre, University of Technology Sydney (UTS), NSW 2007, Australia. they also have some drawbacks such as high system cost with a large number of array channels as well as potential risk of large active standing wave ratio [5]. The other way is to develop nonuniformly spaced techniques to suppress the level of grating lobes. The latter provides a possibility to design nonuniformly spaced UWB arrays with much larger electric element spacings than those used in the tightly coupled antenna arrays. As a result, the design of UWB arrays allow for easy design of antenna array hardware. Nevertheless, they may require relatively large space. Which kind of UWB arrays is better depends on the specific circumstance.

This work focuses on improving the design of nonuniformly spaced UWB arrays. In the literature, many nonuniformly spaced array synthesis methods have been presented. They include, for example, analytical element positioning methods [6], [7], matrix pencil method and its variants [8], [9], compressive sensing (CS) techniques [10]-[13], stochastic optimization-based synthesis techniques [14][18], and some other techniques [19]-[22]. However, most of them deal only with narrow-band sparse array synthesis problems. Direct extension of narrow-band array designs to a wideband case may lead to grating lobes appearing in visible region. In addition, beamscanning can also shift grating lobes into the visible region, which can be equivalent to the consequence of increasing the bandwidth. Hence, the problem of designing element positions to obtain a beamscanning UWB array with considerably reduced grating lobe level (GLL) is much more challenging than the design in the narrowband case. In [23]-[25], Werner and his group have presented several efficient element positioning methods for multi-band or UWB arrays. In particular, an analytical method based on raised power series (RPS) representation has been presented in [25], which can produce an array allocation with a reduced and stable GLL across an ultra-broad frequency band.

Despite its effectiveness and efficiency, the RPS array design relies on analytical symmetrical element positions which would be far from the best. Consequently, the maximum GLL/sidelobe level (SLL) is usually not acceptable in practice for moderate-size RPS arrays. Further optimizing the element positions without the symmetrical distribution assumption would benefit the array performance. In this work, we present a novel method to design beam-scannable UWB arrays. In this method, a concept of design frequency is introduced which transforms the beam-scannable UWB array design to be a broadside-beam single-frequency array design problem. The RPS representation is adopted to generate initial element positions, and then an iterative convex optimization is applied to successively optimize the element positions for further SLL reduction. Multiple constraints for controlling the first-order Taylor expansion accuracy, the minimum element spacing and the array aperture, are all incorporated into the iterative convex optimization to obtain stable and practical synthesis results. It should be noted that although an iterative convex optimization was adopted in [21] to optimize the element positions, the technique in [21] deals only with the fixed broadside beam array design at a single working frequency and it starts the optimization from an uniformly spaced initial array with the spacing that is usually around half a wavelength. In contrast, the 
proposed method deals with the beam-scannable UWB array design where the element positions are optimized at the design frequency that is even much higher than the highest frequency of the working band. The element spacings are much larger than one wavelength at the design frequency. In this situation, starting from a uniformlyspaced array with very high-grating lobes is usually hard to give acceptable optimization result. In addition, the technique in [21] does not constrain the minimum element spacing which is, however, very essential in UWB array design. Several beam-scannable UWB array designs are presented to validate the effectiveness and advantages of the proposed method. The comparisons with the original RPS design technique and some typical stochastic optimization methods are also given in the examples.

\section{Formulation And Algorithms}

\section{A. Beam-scannable UWB array synthesis problem}

Consider a $2 N+1$-element linear array whose elements are located at $\left[z_{-N}, z_{-N+1}, \cdots, z_{N}\right]$ along with $z$-axis. Assume that this array works at the frequency band from $f_{L}$ to $f_{H}$, with a focused beam scanned within the range of $\left[\pi / 2-\theta_{\max }, \pi / 2+\theta_{\max }\right]$. The array factor can be written as the following

$$
A F(\beta, u)=\sum_{n=-N}^{N} \exp \left(j \beta z_{n} u\right)
$$

where $\beta=2 \pi f / c, u=\cos \theta-\cos \theta_{0}$, and $\theta_{0} \in[\pi / 2-$ $\left.\theta_{\max }, \pi / 2+\theta_{\max }\right]$ is the beam pointing direction. Usually, $z_{n}$ is not necessarily equal to $-z_{-n}$ for a general asymmetrical array. However, in particular case, we have $z_{n}=-z_{-n}$ for a symmetrical array. In this situation, the array factor can be rewritten as

$$
A F(\beta, u)=1+2 \sum_{n=1}^{N} \cos \left(\beta z_{n} u\right)
$$

For a UWB array, the highest-to-lowest frequency ratio (HLFR) can be defined as $R_{f}=f_{H} / f_{L}$. In addition, since $\theta_{0} \in[\pi / 2-$ $\left.\theta_{\max }, \pi / 2+\theta_{\max }\right]$, we have $u \in\left[-1-\left|\sin \theta_{\max }\right|, 1+\left|\sin \theta_{\max }\right|\right]$. To evaluate the increment of $u$-region going into visible space due to the beam scanning, we introduce a concept of beam-scanning range ratio (BSRR) which is given by $R_{u}=\max \{u\} / \max \left\{u_{0}\right\}=(1+$ $\left.\left|\sin \theta_{\max }\right|\right)$ where $u_{0}=\cos \theta \in[-1,1]$ denotes the visible $u$-region for a fixed broadside beam. From (1) and (2), the range of the beamscannable UWB array factor has the following property

$$
\begin{gathered}
\left\{A F\left(\beta_{L},[u]\right)\right\}=\left\{A F\left(\beta_{L},\left[R_{u} u_{0}\right]\right)\right\}=\left\{A F\left(R_{u} \beta_{L},\left[u_{0}\right]\right)\right\} \\
\left\{A F\left(\beta_{H},[u]\right)\right\}=\left\{A F\left(R_{f} \beta_{L},\left[R_{u} u_{0}\right]\right)\right\}=\left\{A F\left(R_{f} R_{u} \beta_{L},\left[u_{0}\right]\right)\right\}
\end{gathered}
$$

where $\beta_{H}=2 \pi f_{H} / c, \beta_{L}=2 \pi f_{L} / c$, and $\{A F(\beta,[x])\}$ denotes the range of the function $A F(\beta, x)$ varying with $x \in[x]$. This means that the design of a beam-scannable UWB array can be equivalent to design of a fixed broadside UWB array with much larger HLFR that is increased from $R_{f}$ to $R_{f} R_{u}\left(R_{u} \geq 1\right)$.

\section{B. New beam-scannable UWB array design methodology}

It has been shown in [25] that the array with element positions based on RPS representation has a reduced SLL and GLL over bandwidth of many octaves. However, the analytical RPS positions are not the best and they can be further optimized by some numerical optimization algorithms. One problem associated with application of numerical algorithms to beam-scannable UWB array design is the increased computational and storage loads for dealing with requirements of multiple beam scanning angles at multiple frequencies.
To solve this problem, the proposed idea is introducing a concept of design frequency which transforms the beam-scannable UWB array problem as synthesizing a broadside beam array at the design frequency. Then a two-stage design strategy is developed: at first the RPS representation is adopted to provide good initial element positions, and then an iterative convex optimization with multiple constraints is presented to successively update the element positions until the beam performance reaches to convergence.

1) The concept of design frequency: In general, for the beamscannable UWB array design problem, one should check the scannable beam performance in the whole frequency band of interest. However, as mentioned above, this will significantly increase the computational and storage cost. Fortunately, from (1)-(4) we have that $\left\{A F\left(R_{f} R_{u} \beta_{L},\left[u_{0}\right]\right)\right\}=\left\{A F(\beta, u) \mid \beta \in\left[\beta_{L}, \beta_{H}\right], u \in\right.$ $\left.\left[-R_{u}, R_{u}\right]\right\}$. This indicates that the array factor at the frequency of $f_{D}=R_{u} R_{f} f_{L}$ has included all the beam characteristics of the UWB array in the whole frequency band $\left[f_{L}, f_{H}\right]$ for all beam scanning cases for $\theta_{0} \in\left[\pi / 2-\theta_{\max }, \pi / 2+\theta_{\max }\right]$. Thus we can consider only designing the array factor at the frequency $f_{D}$ to check all the beam performance of a scannable UWB array provided that mutual coupling effect can be ignored. We call this $f_{D}=R_{u} R_{f} f_{L}$ as the design frequency that is not within the working frequency band but a frequency at which we design and check the array factor performance. Such processing allows us to deal with the beam-scannable UWB array design to be like a fixed-beam array factor design at a singlefrequency in some aspects. However, it should be noted that design of the array factor $A F\left(\beta_{D}, u_{0}\right)$ remains to face the same problem in the grating lobe and sidelobe reduction as the design of the original beam-scannable UWB array, since the minimum element spacing at the frequency $f_{D}$ can be multiple times of wavelengths.

2) Initial UWB array design based on RPS representation: The RPS representation is adopted to generate initial element positions for the proposed beam-scannable UWB array design. The RPS positions are given by the following formula [25]

$$
z_{n}=\operatorname{sign}(n) d_{\min } \zeta|n|^{r}, \text { for }|n| \leq N
$$

where $d_{\min }$ is the minimum element spacing, and $\operatorname{sign}(\cdot)$ is a symbolic function which gives $\operatorname{sign}(n)=-1$ for $n<0$ and $\operatorname{sign}(n)=1$ for $n>0$. The parameter $\zeta$ is given by

$$
\zeta(r, N)= \begin{cases}{\left[N^{r}-(N-1)^{r}\right]^{-1},} & 0<r<1 \\ 1, & r \geq 1\end{cases}
$$

From (5) and (6), the RPS positions are non-periodically but symmetrically distributed. The element spacing is given by

$$
\begin{aligned}
d_{n} & =d_{\min } \zeta\left(|n|^{r}-(|n|-1)^{r}\right) \\
& = \begin{cases}d_{\min } \frac{|n|^{r}-(|n|-1)^{r}}{N^{r}-(N-1)^{r}}, & 0<r<1 \\
d_{\min }\left(|n|^{r}-(|n|-1)^{r}\right), & r \geq 1\end{cases}
\end{aligned}
$$

From the above, we can see that the element spacing is actually controlled by the parameters $r, d_{\min }$ and $N$. When $0<r<1$, the spacing $d_{n}$ decreases with the element index $n$, and the minimum spacing $d_{\text {min }}$ is obtained when $|n|=N$. On the contrary, when $r>1, d_{n}$ increases with $n$ and the minimum spacing $d_{\text {min }}$ happens at $|n|=1$. In particular, when $r=1$, the element spacing in (7) is $d_{n}=d_{\min }$ for all $n \leq N$ and the RPS array is reduced to a uniformly spaced array with a spacing of $d_{\text {min }}$. For most of UWB array synthesis cases, the minimum spacing $d_{\min }$ and the element count $N$ can be determined by considering the antenna element structure and gain requirement in applications. The parameter $r$ can be adjusted to improve the beam pattern performance of the initial RPS array. Nevertheless, as mentioned in the introduction, the analytical RPS positions are not the best, and they can further optimized by using 
some numerical iterative optimization strategies. In the following, we will introduce an iterative convex optimization method to significantly improve the wideband beam performance of the original RPS array.

3) Improved $U W B$ array by iterative convex optimization: In order to achieve better UWB array performance, an iterative convex optimization is developed to successively optimize the initial RPS element positions. The basic idea is introducing position shift variables $\delta_{n}$ to the original positions $z_{n}(n=-N, \cdots, N)$ given by (5), and then find a way to obtain the optimal $\delta_{n}$. As mentioned previously, such optimization can be done only for the array factor at the design frequency $f_{D}$. Consider the array factor $A F_{p}\left(\beta_{D}, u_{0}\right)$ associated with perturbed positions $z_{n}+\delta_{n}(n=-N, \cdots, N)$. That is given by

$$
A F_{p}\left(\beta_{D}, u_{0}\right)=\sum_{n=-N}^{N} \exp \left\{j \beta_{D}\left(z_{n}+\delta_{n}\right) u_{0}\right\}
$$

Then applying the first-order Taylor expansion of $e^{j \beta_{D} \delta_{n} u_{0}} \approx 1+$ $j \beta_{D} \delta_{n} u_{0}$ for the case of $\left|\beta_{D} \delta_{n} u_{0}\right| \ll 1$ to the above expression, we can obtain

$$
A F_{p}\left(\beta_{D}, u_{0}\right) \approx A F\left(\beta_{D}, u_{0}\right)+j \beta_{D} u_{0} \sum_{n=-N}^{N} \delta_{n} \exp \left(j \beta_{D} z_{n} u_{0}\right)
$$

where $A F\left(\beta_{D}, u_{0}\right)$ is the original array factor with element positions $z_{n}$. Now, the shift variables $\delta_{n}$ after the first-order Taylor approximation behaviors like weight coefficients which can be optimized by using convex optimization to further reduce the overall SLL. However, for a practical UWB array design problem, the following three kinds of constraints should be considered:

a) First-order Taylor expansion accuracy constraint. The effectiveness of (9) depends on the accuracy of the first-order Taylor expansion. To guarantee the effectiveness, it is required that $\left|\beta_{D} \delta_{n} u_{0}\right| \ll 1$. Since $\left|u_{0}\right| \leq 1$, we can set that $\left|\beta_{D} \delta_{n}\right| \leq \mu$ where $\mu$ is a parameter used to balance the allowable maximum position shift $\delta_{\max }=\mu / \beta_{D}$ and the Taylor expansion accuracy. b) The minimum element spacing constraint. In application, the minimum element spacing constraint is very important from the perspectives of antenna element structure design and mutual coupling reduction. This constraint should be maintained in the convex optimization process. That is, the new positions should satisfy the constraint of $\left(z_{n}+\delta_{n}\right)-\left(z_{n-1}+\delta_{n-1}\right) \geq d_{\min }$ for all $n=-N+1, \cdots, N$, where $\delta_{-N}=0$.

c) The array aperture constraint. The array aperture should be constrained when the available space is limited. This constraint can be dealt as $\left(z_{N}+\delta_{N}\right)-\left(z_{-N}+\delta_{-N}\right) \leq A$ where $A$ is the maximum value of acceptable array aperture.

Thus, the optimal position shift variables can be found by solving the following constrained optimization problem

$\min \epsilon$

$$
\text { s.t. }\left\{\begin{array}{l}
\left|A F\left(\beta_{D}, u_{0}^{s}\right)+j \beta_{D} u_{0}^{s} \sum_{n=-N}^{N} \delta_{n} e^{j \beta_{D} z_{n} u_{0}^{s}}\right| \leq \epsilon, u_{0}^{s} \in U_{S L} ; \\
\left(z_{n}+\delta_{n}\right)-\left(z_{n-1}+\delta_{n-1}\right) \geq d_{\min }, n=-N+1, \cdots, N ; \\
\left(z_{N}+\delta_{N}\right)-\left(z_{-N}+\delta_{-N}\right) \leq A ; \\
\delta_{-N}=0 \\
\left|\beta_{D} \delta_{n}\right| \leq \mu, n=-N, \cdots, N
\end{array}\right.
$$

where $U_{S L}$ denotes the sidelobe region in $u_{0}$-space. Clearly, the above problem can be efficiently solved by using convex optimization. It should be noted that the position shifts obtained in this way
Algorithm 1 The proposed beam-scannable UWB array design procedure

1: Set the frequency band $\left[f_{L}, f_{H}\right]$ and beam scanning range $[\pi / 2-$ $\left.\theta_{\max }, \pi / 2+\theta_{\max }\right]$, and then calculate the highest-to-lowest frequency ratio $R_{f}$, the beam scanning range ratio $R_{u}$ and the design frequency $f_{D}$.

2: Set the minimum element spacing $d_{\min }$, element number parameter $N$ (the total number is $2 N+1$ ), and the allowable maximum array aperture $A$ (if it exists), all depending on the application requirement.

3: Apply the RPS representation (5)-(7) to generate initial element positions $z_{n}$ for $n=-N, \cdots, N$ with an appropriate $r$. The paramter $r$ can be chosen such that the obtained RPS array has the lowest maximum SLL at $f_{D}$ among different $r$ and the array aperture $2 d_{\min } \zeta N^{r}$ should be no larger than $A$.

4: Set $k=0$. Sample the space of $u_{0}$ and initialize the parameter $\mu$ for allowable maximum shift $\delta_{\max }$.

5: $k=k+1$.

6: Solve the constrained convex optimization problem (10) to find the best position shifts $\delta_{n}(n=-N, \cdots, N)$.

7: Update the element positions by $z_{n}=z_{n}+\delta_{n}(n=-N, \cdots, N)$.

8: Compute the pattern $A F_{p}\left(\beta_{D}, u_{0}\right)$ in (8) with the new positions, and calculate the maximum SLL $S L L_{\max }$ of this pattern.

9: Repeat Step 5 to 9 until $S L L_{\max }$ remains the same for multiple times or $k$ reaches the allowable maximum number of iterations.

10: Output the obtained element positions $z_{n}(n=-N, \cdots, N)$ and calculate the beam-scannable wideband pattern $A F(\beta, u)$ in (1).

are optimal under the given constraints, but due to the limitation of $\left|\beta_{D} \delta_{n}\right| \leq \mu$ for accurate first-order Taylor expansion, the allowable maximum position shifts $\delta_{\max }$ are very electrically small so that the achievable SLL reduction is limited. Hence, an iterative convex optimization strategy can be developed in which each iteration finds locally optimal position shifts and thus the element positions can be successively updated until the obtained SLL maintains the same for many times. Besides, it should be mentioned that since all the excitation amplitudes remain ones, the beam direction of the array factor always keeps at $\theta=0^{\circ}$ in the position perturbation optimization process.

\section{The proposed beam-scannable UWB array design procedure}

The proposed beam-scannable UWB array design procedure is given in Algorithm 1. In this procedure, the design frequency $f_{D}$ is determined at first and then the problem is transformed as designing a broadside fixed-beam array at $f_{D}$. The RPS representation is adopted to generate initial element positions. The parameter $r$ should be appropriately selected such that the achievable maximum SLL for the initial array is as low as possible and the array aperture meets its allowable limit if it has. Then a number of constrained convex optimizations given in (10) are performed to successively update the element positions such that the obtained maximum SLL can be reduced as much as possible.

\section{NumERICAL RESUlTS}

In this section, several beam-scannable UWB arrays with different settings in HLFR, BSRR and array size, are presented to show the effectiveness and robustness of the proposed method. Parameters study is included to show the guideline of applying this method. The comparisons with the original RPS design and other optimization methods are also provided.

\section{A. 65-element beam-scannable UWB array design}

As the first example, we consider synthesizing a UWB array occupying the frequency band from 2 to $6 \mathrm{GHz}$ with beam scanning 
from $45^{\circ}$ to $135^{\circ}$. That is, $R_{f}=3$ and $R_{u}=1+\sin \left(45^{\circ}\right)=1.707$. Thus, we can set the design frequency as $f_{D}=R_{u} R_{f} f_{L} \approx 5.12$ GHz. The minimum spacing constraint is set as $d_{\text {min }}=0.5 \lambda_{L}$ at $2 \mathrm{GHz}$, which is equal to $2.56 \lambda_{D}$ at $f_{D}$. Set $N=32$ which corresponds to a 65 -element array. In this example, we assume there is no aperture constraint. In the first stage, we apply the RPS representation to generate the initial element positions, and the parameter $r$ is varied from 0.75 to 1.25 in step of 0.01 to find the best initial positions in terms of reducing the maximum SLL. Fig. 1 shows the maximum SLL of the RPS array with different $r$ at the design frequency $f_{D}$ which is actually equal to the overall maximum SLL across the whole band $\left[f_{L}, f_{H}\right]$ for the beam scanning within $\left[45^{\circ}, 135^{\circ}\right]$. As can be seen, the parameter $r$ affects the maximum SLL of the RPS array. Within the range of $r \in[0.75,1], r=0.77$ gives the lowest maximum SLL of $-8.46 \mathrm{~dB}$, and within the range of $r \in[1,1.25], r=1.25$ gives the lowest maximum SLL of -9.12 $\mathrm{dB}$. On the other hand, different $r$ also significantly affects the total aperture of the array, as shown in Fig. 1. The minimum aperture happens at $r=1$ which corresponds to the case of a uniformly spaced array with a spacing of $d_{\min }=\lambda_{L} / 2=2.56 \lambda_{D}$. Since $\left.d_{\text {min }} / \lambda_{D}\right) \geq 1$, this uniformly spaced array has grating lobes.

Now, we apply the iterative convex optimization in the second stage of the proposed method to successively optimize the element positions and suppress the maximum SLL. To study the affect of the allowable maximum position shift $\delta_{\max }$ in each iteration on the performance of the proposed method, we set $\mu=\pi /[5,10,15,20]$ which corresponds to $\delta_{\max }=\lambda_{D} /[10,20,30,40]$. Fig. 2 shows the SLL reduction of the pattern at $f_{D}$ versus the number of iterations at different $\delta_{\max }$ for different initial RPS positions with (a) $r=0.77$ and (b) $r=1.25$, respectively. As can be seen, the SLL decreases as the number of iterations increases for all the test cases. The smaller $\delta_{\max }$ is, the slower the SLL decreases. However, for the case of $r=0.77$ and $\delta_{\max }=\lambda_{D} / 10$, the SLL convergence seems not always stable due to relatively low accuracy of the first-order Taylor approximation. Usually, selecting around $\lambda_{D} / 20$ for the $\delta_{\max }$ would be reasonable in terms of the balance between stability and efficiency. It should be noted that for the case of $r=0.77$, the SLL is reduced from $-8.46 \mathrm{~dB}$ for the initial RPS array to $-13.99 \mathrm{~dB}$ for the optimized array, and for the case of $r=1.25$, the SLL is reduced from $-9.12 \mathrm{~dB}$ to $-14.50 \mathrm{~dB}$. For instance, Fig. 3(a) and (b) show the wideband patterns of the initial RPS array $(r=0.77)$ with beam pointing at $90^{\circ}$ and $135^{\circ}$, respectively, and Fig. 3(c) and (d) show the corresponding broadside and scanned patterns of the finally optimized array. In addition, the overall maximum SLL and aperture size for the optimized array using different initial RPS positions with different $r$ are also included in Fig. 1 for more comprehensive comparison. We can see that compared to the initial RPS array, the proposed method can significantly reduce the maximum SLL by more than $5 \mathrm{~dB}$ for most of $r \in[0.75,1.25]$ while maintaining almost the same array aperture.

\section{B. 101-element beam-scannable UWB array with aperture limit}

In the second example, we check the effectiveness of the proposed method for designing a UWB array with more elements for larger relative bandwidth and wider beam-scanning range. In addition, the maximum aperture limit is also considered. Assume that the number of elements is $101(N=50)$, the required bandwidth is from 1 to 5.6 $\mathrm{GHz}$, and the beam scanning range is from $38^{\circ}$ to $142^{\circ}$. In this case, $R_{f}=5.6, R_{u}=1+\sin \left(52^{\circ}\right)=1.788$, and the design frequency is $f_{D}=R_{u} R_{f} f_{L} \approx 10 \mathrm{GHz}$. The minimum spacing constraint is set as $d_{\min }=0.5 \lambda_{L}$ at $1 \mathrm{GHz}$, which is equal to $5 \lambda_{D}$ at $f_{D}$. In the first stage, we still adopt the RPS representation to generate the initial element positions. The parameter $r$ is varying from 0.75 to

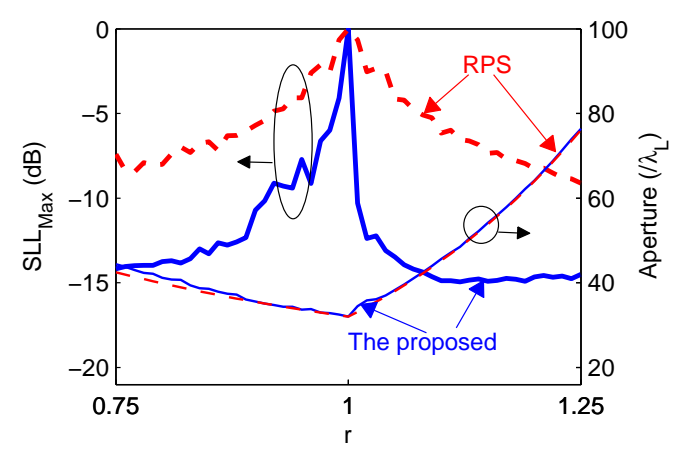

Fig. 1. The maximum SLLs and apertures of the initial 65-element RPS array with different $r \in[0.75,1.25]$ and the optimized array by the proposed method.

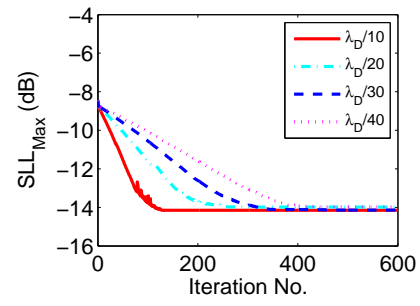

(a)

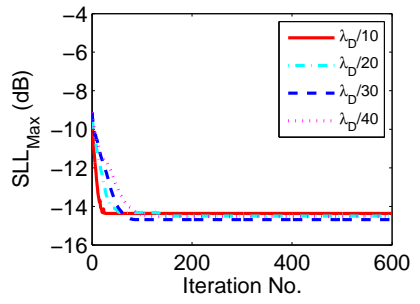

(b)
Fig. 2. The achievable maximum SLL versus the number of iterations at different $\delta_{\max }$ for the 65-element optimized array. Different initial RPS positions are used: (a) $r=0.77$, and (b) $r=1.25$.

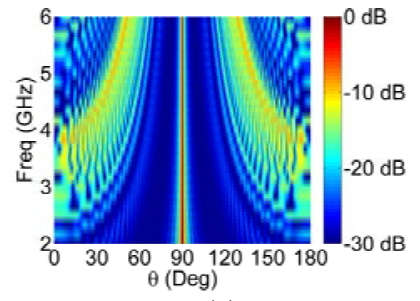

(a)

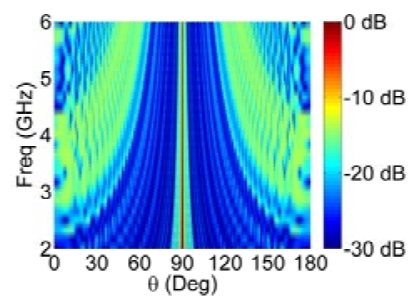

(c)

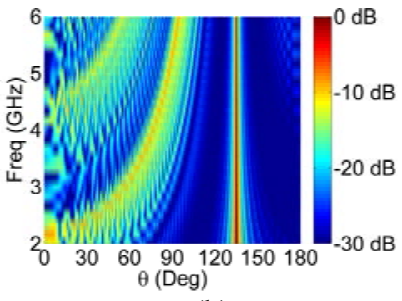

(b)

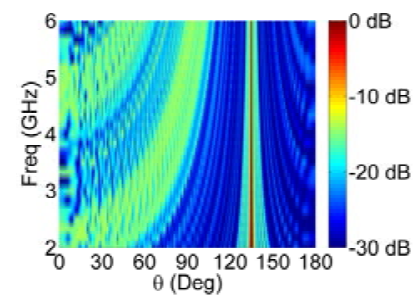

(d)
Fig. 3. Broadside and scanned wideband patterns of the 65-element array with initial RPS positions $(N=32, r=0.77)$ and the proposed optimized array. (a) broadside pattern of the initial RPS array, (b) scanned pattern (beam pointing at $135^{\circ}$ ) of the initial RPS array, (c) broadside pattern of the optimized array, and (d) scanned pattern (beam pointing at $135^{\circ}$ ) of the optimized array.

1.07, so that the obtained aperture for the RPS array is no more than $66.50 \lambda_{L}$, as shown in Fig. 4(a). That is, the maximum aperture is equal to 1.33 times of that for a 101 -element $0.5 \lambda_{L}$-spaced array $(r=1)$. Fig. 4(b) shows the obtained maximum SLL of the RPS array with different $r \in[0.75,1.07]$ at the design frequency $f_{D}$ (this is also the overall maximum SLL of the array across the whole interesting band and beam scanning range). As can be seen, within the range of $r \in[0.75,1], r=0.81$ gives the lowest maximum SLL 


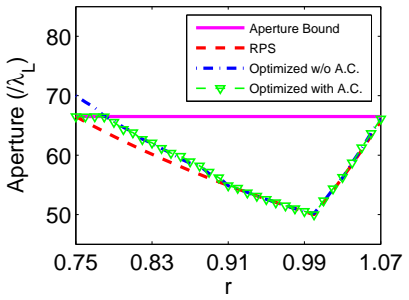

(a)

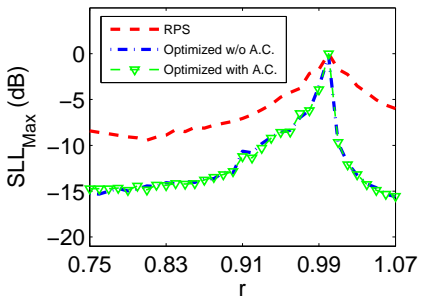

(b)
Fig. 4. (a) The maximum SLLs and (b) apertures of the initial 101-element RPS array with different $r \in[0.75,1.07]$ and the optimized arrays by the proposed method with/without aperture constraint.

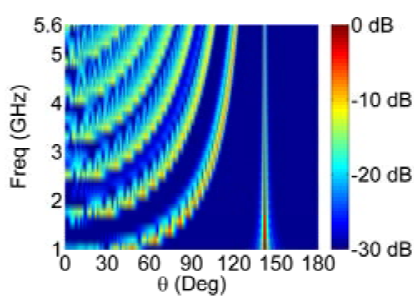

(a)

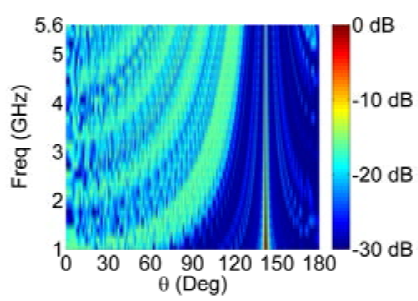

(b)
Fig. 5. The scanned wideband patterns (beam pointing at $142^{\circ}$ ) for the 101-element array with (a) the initial RPS positions $(N=50, r=1.07)$ and (b) the optimized positions with aperture limit.

of $-9.43 \mathrm{~dB}$, while within the range of $r \in[1,1.07], r=1.07$ gives the lowest maximum SLL of $-5.99 \mathrm{~dB}$.

In the second stage, we apply the iterative convex optimization with $\delta_{\max }=\lambda_{D} / 20$ to optimize the element positions for further reducing the maximum SLL. The aperture limit is set as $A=66.50 \lambda_{L}$. The obtained maximum SLL and the aperture of the optimized array under this aperture constraint for different $r$ are shown in Fig. 4. For comparison, the results for the array optimized without aperture limit are included as well. It is clearly seen that both of the optimized arrays with/without the aperture limit have significantly reduced the maximum SLL except for the nearby region of $r=1$. For example, for the case of $r=0.81$, the maximum SLL for the optimized array with aperture limit is reduced from $-9.43 \mathrm{~dB}$ to $-14.85 \mathrm{~dB}$. For the case of $r=1.07$, the maximum SLL obtained with aperture limit is reduced from $-5.99 \mathrm{~dB}$ to $-15.56 \mathrm{~dB}$. In addition, the aperture constraint indeed works for the proposed method. For the case of using initial RPS array with $r \in[0.75,0.78]$, the optimized array without aperture limit has the aperture exceeding the upper bound of $66.50 \lambda_{L}$ while the one with the aperture constraint always meets the bound. Fig. 5(a) shows the scanned wideband pattern with beam pointing at $142^{\circ}$ of the initial RPS array $(r=1.07)$, and Fig. 5(b) show the corresponding scanned pattern of the array optimized with the aperture constraint.

\section{Comparison with other methods}

In the last example, we check the performance of the proposed method in terms of the obtained SLL and time cost for synthesizing different arrays with varying sizes. Four sizes of UWB arrays are considered, and they consist of 51, 101, 151 and 201 elements (i.e., $N=[25,50,75,100])$, respectively. For all the arrays, assume that the interesting frequency band is from 1 to $4 \mathrm{GHz}$ with beam scanning from $45^{\circ}$ to $135^{\circ}$. That is, $R_{f}=4, R_{u}=1+\sin \left(45^{\circ}\right)=1.707$ and $f_{D}=R_{u} R_{f} f_{L} \approx 6.83 \mathrm{GHz}$. The minimum spacing constraint is set as $d_{\text {min }}=0.5 \lambda_{L}$ at $1 \mathrm{GHz}$. In addition, assume that the allowable maximum aperture for each size of array is 1.4 times of that for a $0.5 \lambda_{L}$-spaced array with the same number of elements. The RPS representation with an appropriate $r>1$ is chosen such that the obtained SLL for the initial array reaches the minimum under the condition that the aperture meets its limitation. The obtained parameter $r$, SLL, aperture and averaging spacing of the initial RPS array for each array size case is shown in Table I. Then, we perform the second stage of the proposed method (with $\delta_{\max }=\lambda_{D} / 20$ ) to successively optimize the element positions for further SLL reduction. In addition, for a more comprehensive comparison, some stochastic optimization algorithms including genetic algorithm (GA) and particle swarm optimization (PSO) are also applied to synthesize these arrays, with the same configurations in the frequency band, scanning range, minimum spacing and aperture constraint. The concept of design frequency is also used in the both stochastic optimization algorithms for fair comparison. For the GA optimization, the population sizes are set as 250, 500, 750, and 1000 for the arrays with 51, 101, 151, and 201 elements, respectively. The number of generations is set as 5000. For the PSO method, the population sizes are set to be 70 , 140, 210, and 280 for the 51, 101, 151, and 201-element arrays, respectively. Due to the randomness of some operations in either GA or PSO, synthesis results obtained from different runs may not be identical. So, we perform the synthesis procedure 5 times for both GA and PSO, and pick up the lowest SLL as the final result. The time cost is calculated as the average over the 5 runs. Table I lists the obtained SLLs and time costs for all the test methods (all the results are obtained on the same computer with Intel(R) Core(TM) i3-4160 CPU@3.60GHz).

As can be seen, under the prescribed aperture constraint and wideband beam scanning requirement, the initial RPS array gives very high SLL for each array size case. The SLL of the RPS array varies from $-5.28 \mathrm{~dB}$ to $-8.50 \mathrm{~dB}$ as the element count increases from 51 to 201. Such SLL performances are not satisfactory in most of applications. Nevertheless, the RPS representation provides good initial element positions, and after further optimization based on these initial positions, the proposed method obtains a much lower SLL for each case. Compared with the results of the initial RPS arrays, the SLL reduction by the proposed method is more than $7.9 \mathrm{~dB}$ for all the test cases shown in Table I. The GA and PSO achieve almost the same SLL performance as that of the proposed method only for the 51-element UWB array case. However, for the case of larger size arrays (with 101, 151 and 201 elements, respectively), both GA and PSO have much higher SLLs than those obtained by the proposed method. Especially for the case of 201element UWB array, the obtained SLLs for the GA and PSO (5 times of implementations are run for each case) are more than $4 \mathrm{~dB}$ higher than that of the proposed method. This shows that although these stochastic algorithms can potentially find the globally optimum solution, in actual implementation they may be hard to reach the best, with limited population size and generations. This issue becomes more challenging in large-scale antenna array optimization problems (e.g., the arrays with 100 elements or even more). Besides, although the time cost required by the proposed method increases considerably with the number of elements, it is still much less than that of either GA or PSO for each test case.

To further evaluate the performance of the proposed method, we check the result of the iterative convex optimization using an initial array with randomly distributed element positions. For example, we consider the 101-element array case. Assume that the initial element spacings are set as uniform random numbers within $[2,3.48] \lambda_{H}$ (the averaging element spacing is about $2.73 \lambda_{H}$ ). Other configurations such as frequency band, beam scanning angle, minimum spacing constraint are set as the same as those in Table I. We run the same optimization procedure 30 times with different initial random arrays. The obtained SLLs for the optimized arrays with different random initial arrays are ranging from -13.06 to $-16.28 \mathrm{~dB}$. This means 
TABLE I

THE SLLS AND TIME COSTS OF DIFFERENT METHODS FOR SYNTHESIZING DIFFERENT SIZES OF BEAM-SCANNABLE UWB ARRAYS $\left(R_{f}=4\right.$, $\left.R_{u}=1.707, d_{\min }=0.5 \lambda_{L}, \delta_{\max }=\lambda_{D} / 20\right)$.

\begin{tabular}{|c|c|c|c|c|c|c|c|c|c|c|c|c|c|}
\hline \multirow{2}{*}{$\begin{array}{l}\text { No. of } \\
\text { Elem. }\end{array}$} & \multirow[t]{2}{*}{$r$} & \multirow[t]{2}{*}{$N$} & \multirow{2}{*}{$\begin{array}{c}\mathrm{FNBW}\left({ }^{\circ}\right) \\
\text { at } f_{L}\end{array}$} & \multirow{2}{*}{$\begin{array}{l}\text { Aperture } \\
\left(/ \lambda_{L}\right)\end{array}$} & \multirow{2}{*}{$\begin{array}{l}\text { Aver. Spacing } \\
\qquad\left(/ \lambda_{H}\right)\end{array}$} & \multicolumn{4}{|c|}{$S L L_{\mathrm{Max}}(\mathrm{dB})$} & \multicolumn{4}{|c|}{ Time cost ${ }^{*}$} \\
\hline & & & & & & RPS & GA & PSO & Proposed & RPS & GA & PSO & Proposed \\
\hline 51 & 1.10 & 25 & 3.47 & 34.49 & 2.76 & -5.28 & -12.84 & -13.03 & -13.19 & $40.2 \mu \mathrm{s}$ & $2.36 \mathrm{~h}$ & $1.31 \mathrm{~h}$ & $0.09 \mathrm{~h}$ \\
\hline 101 & 1.08 & 50 & 1.75 & 68.37 & 2.73 & -6.49 & -13.51 & -13.84 & -16.12 & $45.6 \mu \mathrm{s}$ & $16.60 \mathrm{~h}$ & $9.46 \mathrm{~h}$ & $2.37 \mathrm{~h}$ \\
\hline 151 & 1.07 & 75 & 1.16 & 101.46 & 2.71 & -7.48 & -14.45 & -14.53 & -17.99 & $51.9 \mu \mathrm{s}$ & $54.59 \mathrm{~h}$ & $31.08 \mathrm{~h}$ & $19.34 \mathrm{~h}$ \\
\hline 201 & 1.07 & 100 & 0.87 & 138.04 & 2.76 & -8.50 & -15.06 & -14.95 & -19.11 & $56.5 \mu \mathrm{s}$ & $132.21 \mathrm{~h}$ & $72.82 \mathrm{~h}$ & $35.47 \mathrm{~h}$ \\
\hline
\end{tabular}

* For either GA or PSO method, the total time cost for 5 runs to pick up the lowest SLL is 5 times of the cost listed above.

that compared with the $-16.12 \mathrm{~dB}$ SLL obtained by the optimization with the RPS initial array, the lowest SLL among 30 times of runs for the random initial arrays is only slightly lower, while the worst result has much higher SLL. In addition, for 30 times of runs, there are 27 times when the optimization using RPS initial array outperforms the optimization using random initial array. This further validates the advantage of the proposed iterative convex optimization based on the RPS initial array.

\section{CONCLUSION}

By virtue of the concept of design frequency, the proposed method transforms the beam-scannable UWB design to a problem of synthesizing a broadside fixed-beam array at a single-frequency. The RPS representation with an appropriate parameter choice is used to provide good initial element positions (with reduced grating lobes), and then the iterative convex optimization is applied to successively optimize the positions for further SLL reduction. In addition, the minimum element spacing constraint and aperture limitation can be easily incorporated in the proposed synthesis procedure so that the obtained array configuration can be implemented in practice. Synthesis results show that the proposed method achieves much lower SLLs than those by the original RPS method for all test cases, and it also significantly outperforms the conventional GA and PSO methods for large size of beam-scannable UWB arrays (i.e., the ones with more than 100 elements).

Finally, it should be noted that the design of beam-scannable UWB arrays with low SLL is recognized as a very challenging problem due to the fact that the element spacings are usually much more than one wavelength at higher frequency of the band. The proposed method provides an effective and robust solution to deal with this problem.

\section{REFERENCES}

[1] D. W. O’Hagan, M. Schroder, V. Basavarajappa, P. Knott, H. Kuschel, and M. Simeoni, "Wideband antenna array for digital video broadcast terrestrial-based passive bistatic radar applications," IET Radar Sonar Navig., vol. 8, no. 2, pp. 106-113, Feb. 2014.

[2] M. T. Ghasr, M. J. Horst, M. R. Dvorsky, and R. Zoughi, "Wideband microwave camera for real-time 3-D imaging," IEEE Trans. Antennas Propag., vol. 65, no. 1, pp. 258-268, Jan. 2017.

[3] I. Tzanidis, K. Sertel, and J. L. Voakis, "UWB low-profile tightly coupled dipole array with integrated balun and edge terminations," IEEE Trans. Antennas Propag., vol. 61, no. 6, pp. 3017-3025, Jun. 2013.

[4] H. Zhang, S. Yang, Y. Chen, J. Guo, and Z. Nie, "Wideband dual-polarized linear array of tightly coupled elements," IEEE Trans. Antennas Propag., vol. 66, no. 1, pp. 476-480, Jan. 2018.

[5] Y. Chen, S. Yang, and Z. Nie, "A novel wideband antenna array with tightly coupled octagonal ring elements," Prog. Electromagn. Res., vol. 124, pp. 55-70, Jan. 2012.

[6] Y. Lo and S. Lee, "A study of space-tapered arrays," IEEE Trans. Antennas Propag., vol. 14, no. 1, pp. 22-30, Jan. 1966.

[7] B. P. Kumar and G. R. Branner, "Generalized analytical technique for the synthesis of unequally spaced arrays with linear, planar, cylindrical or spherical geometry," IEEE Trans. Antennas Propag., vol. 53, no. 2, pp. 621-634, Feb. 2005.
[8] Y. Liu, Z. Nie, and Q. H. Liu, "Reducing the number of elements in a linear antenna array by the matrix pencil method," IEEE Trans. Antennas Propag., vol. 56, no. 9, pp. 2955-2962, Sep. 2008.

[9] H. Shen and B. Wang, "An effective method for synthesizing multiplepattern linear arrays with a reduced number of antenna elements," IEEE Trans. Antennas Propag., vol. 65, no. 5, pp. 2358-2366, May. 2017.

[10] G. Oliveri, M. Carlin, and A. Massa, "Complex-weight sparse linear array synthesis by Bayesian compressive sampling," IEEE Trans. Antennas Propagat., vol. 60, no. 5, pp. 2309-2326, May 2012.

[11] G. Prisco and M. D'Urso, "Maximally sparse arrays via sequential convex optimizations," IEEE Antennas Wireless Propag. Lett., vol. 11, pp. 192-195, Mar. 2012.

[12] F. Viani, A. Oliveri, and A. Massa, "Compressive sensing pattern matching techniques for synthesizing planar sparse arrays," IEEE Trans. Antennas Propag., vol. 61, no. 9, pp. 4577-4587, Jul. 2013.

[13] A. F. Morabito, A. R. Lagana, G. Sorbello, and T. Isernia, "Maskconstrained power synthesis of maximally sparse linear arrays through a compressive-sensing-driven strategy," J. Electromagn. Waves Appl., vol. 29, no. 10, pp. 1384-1396, 2015.

[14] R. L. Haupt, "Thinned arrays using genetic algorithms," IEEE Trans. Antennas Propag., vol. 42, no. 7, pp. 993-999, Jul. 1994.

[15] F. J. Ares-Pena, J. A. Rodriguez-Gonzalez, E. Villanueva-Lopez, and S. R. Rengarajan, "Genetic algorithms in the design and optimization of antenna array patterns," IEEE Trans. Antennas Propag., vol. 47, no. 3, pp. 506-510, Mar. 1999.

[16] D. G. Kurup, M. Himdi, and A. Rydberg, "Synthesis of uniform amplitude unequally spaced antenna arrays using the differential evolution algorithm," IEEE Trans. Antennas Propag., vol. 51, no. 9, pp. 2210-2217, Sep. 2003.

[17] A. Modiri and K. Kiasaleh, "Modification of real-number and binary PSO algorithms for accelerated convergence," IEEE Trans. Antennas Propag., vol. 59, no. 9, pp. 214-224, Jan. 2011.

[18] R. Bhattacharya, T. K. Bhattacharyya, and R. Garg, "Position mutated hierarchical particle swarm optimization and its application in synthesis of unequally spaced antenna arrays," IEEE Trans. Antennas Propag., vol. 60, no. 7, pp. 3174-3181, Jul. 2012.

[19] J. I. Echeveste, M. Á. González de Aza, J. Rubio, and C. Craeye, "Gradient-based aperiodic array synthesis of real arrays with uniform amplitude excitation including mutual coupling," IEEE Trans. Antennas Propag., vol. 65, no. 2, pp. 541-551, Feb. 2017

[20] P. You, Y. Liu, S. Chen, K. D. Xu, W. Li, and Q. H. Liu, "Synthesis of unequally spaced linear antenna arrays with minimum element spacing constraint by alternating convex optimization," IEEE Antennas Wireless Propag. Lett., vol. 16, pp. 3126-3130, Oct. 2017.

[21] B. Fuchs, A. Skrivervik, and J. R. Mosig, "Synthesis of uniform amplitude focused beam arrays," IEEE Antennas Wireless Propag. Lett., vol. 11, pp. 1178-1181, Oct. 2012.

[22] H. V. Bui, S. N Jha, and C.Craeye, "Fast full-wave synthesis of printed antenna arrays including mutual coupling," IEEE Trans. Antennas Propag., vol. 64, no. 12, pp. 5163-5171, Dec. 2016.

[23] D. H. Werner, M. A. Gingrich, and P. L. Werner, "A self-similar fractal radiation pattern synthesis technique for reconfigurable multiband arrays ," IEEE Trans. Antennas Propag., vol. 51, no. 7, pp. 1486-1498, Jul. 2003.

[24] T. G. Spence, D. H. Werner, and J. N. Carvajal, "Modular broadband phased-arrays based on a nonuniform distribution of elements along the Peano-Gosper space-filling curve," IEEE Trans. Antennas Propag., vol. 58, no. 8, pp. 600-604, Feb. 2010.

[25] M. D. Gregory and D. H. Werner, "Ultrawideband aperiodic antenna arrays based on optimized raised power series representations," IEEE Trans. Antennas Propag., vol. 58, no. 3, pp. 756-764, Mar. 2010. 